\title{
On the Equilibrium of Simplicial Masonry Structures
}

\author{
Fernando de Goes \\ Caltech
}

\author{
Houman Owhadi \\ Caltech
}

\author{
Mathieu Desbrun \\ Caltech
}

\begin{abstract}
We present a novel approach for the analysis and design of selfsupporting simplicial masonry structures. A finite-dimensional formulation of their compressive stress field is derived, offering a new interpretation of thrust networks through numerical homogenization theory. We further leverage geometric properties of the resulting force diagram to identify a set of reduced coordinates characterizing the equilibrium of simplicial masonry. We finally derive computational form-finding tools that improve over previous work in efficiency, accuracy, and scalability.
\end{abstract}

Keywords: architectural geometry, discrete differential geometry, geometry processing, orthogonal reciprocal diagram.

Links: DL 国PDF WEB

\section{Introduction}

The most subtle and exquisite part of architecture [...] is the formation
of [...] vaults; cutting their stones, and adjusting them with such artifice,
that the same gravity and weight that should have precipitated them to
the ground, maintain them constantly in the air, supporting one another
in virtue of the mutual complication which binds them [...].
Vicente Tosca, Compendio Matematico (vol. 5-15), 1727

Masonry structures are arrangements of material blocks, such as bricks or stones, that support their own weight. Constructing curved vaults or domes with compression-only structures of blocks, further prevented from slipping through friction and/or mortar, has been practiced since antiquity. It is therefore no surprise that form finding and stability analysis of masonry structures have been an active area of research for years.

Equilibrium of a masonry structure is ensured if there exists an inner thrust surface which forms a compressive membrane resisting the external loads [Heyman 1966]. Balance conditions relating the stress field on the thrust surface to the loads are well understood in the continuous setting [Giaquinta and Giusti 1985; Fosdick and Schuler 2003; Angelillo et al. 2012]. Discretizing these equations have been done through conforming and non-conforming finite elements, with formulations involving stress, displacements, or both [Fraternali et al. 2002; Fraternali 2010; Fraternali 2011]. The discrete block-based nature of masonry has also led to the analysis of the network of compressive forces keeping masonry blocks together while resisting external loads [Block 2009]. The rich geometry of this force diagram has received attention from the geometry processing community as well, with recent work on the structural soundness of masonry buildings [Whiting et al. 2009; Whiting et al. 2012] and on the design of self-supporting polygonal meshes [Vouga et al. 2012].

In spite of the variety of computational techniques currently available, existing tools still impose stringent limitations on the process

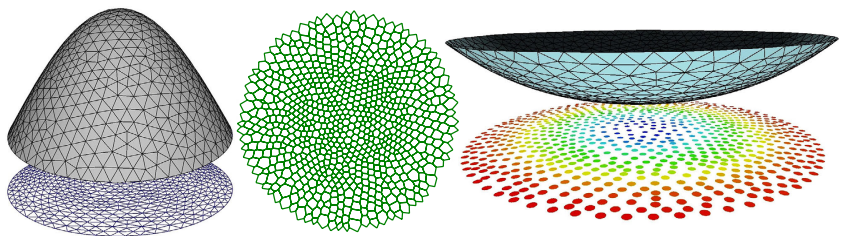

Figure 1: Simplicial Masonry. A self-supporting simplicial masonry structure is a triangle mesh, defined as a height field over the plane (left). We show through numerical homogenization of the stress tensor that they support their own weights if there exists an orthogonal dual diagram (middle), representing a finite-dimensional approximation of the purely compressive forces at play. We provide discrete counterparts to a number of continuous fields (such as the convex Airy stress function, right) and differential equations traditionally used in describing the equilibrium of these masonry buildings; in particular, we offer a set of reduced coordinates of the space of statically admissible shapes, linking our approach to regular triangulations.

of form finding for masonry structures: while previous finite element methods are known to restrict the topology of masonry structures to the case of simply connected domains, thrust network approaches may lead to overconstrained balance equations depending on the choice of boundary conditions. To overcome these issues, we introduce in this paper a discrete theory of simplicial masonry structures. We show that the self-supporting properties of discrete simplicial structures can be derived from a numerical homogenization of the underlying continuous differential equations. By leveraging previous methods, we offer a unified computational framework that enforces the compressive nature and the equilibrium of masonry structures exactly, for surfaces of arbitrary topology. In the process, we introduce reduced coordinates to generate all possible reciprocal force diagrams from simplicial meshes, and reveal geometric connections to well-known continuous notions such as the Airy stress function. Finally, we turn our theoretical contributions into an effective computational technique for the design of simplicial masonry structures that offers improved performance over previous work.

\section{Discrete Self-Supporting Surfaces}

The analysis of masonry structures follows two common assumptions [Heyman 1966; Giaquinta and Giusti 1985]: no tensile strength or material failure is at play; and there exists a thrust surface, contained within the masonry structure, in static equilibrium with the load applied to the structure (including its own weight). A masonry structure based on these conditions is named a self-supporting surface, and we will further concentrate on pure vertical loading. We review next the continuum formulation of self-supporting surfaces, before describing how one can upscale (or homogenize) these equations and turn them into a finite-dimensional problem-which will provide rigorous foundations and extensions to the common use of reciprocal force networks [Block 2009; Vouga et al. 2012].

\subsection{Setup and definitions}

Before explaining our approach, we first fix a few notations.

Continuum Setup. A self-supporting surface $\mathcal{S}$ is henceforth represented as a height function $z$ over a two-dimensional domain $\mathcal{U}$ with a Cartesian coordinate system $\mathbf{u}=[u, v]$ :

$$
[u, v] \rightarrow S(u, v)=[u, v, z(u, v)]
$$

where we use brackets to concisely denote vectors. The Jacobian matrix $\nabla S=\left[\mathbb{I}, \nabla z^{t}\right]$ of this map lifts (i.e., pushforwards) vectors 
from $\mathcal{U} \subset \mathbb{R}^{2}$ to tangent vectors on $\mathcal{S}$ expressed in $\mathbb{R}^{3}$, and thence defines an induced metric tensor $M$ on $\mathcal{S}$ as $M=(\nabla S)^{t} \nabla S$. Consequently, quantities on the surface $\mathcal{S}$ (that we will denote for clarity by an overline ${ }^{-}$) can be expressed in the plane as a function of $u$ and $v$. For instance, the mass density $\bar{\rho}$ at a surface point $S(u, v)$ defines a density function $\rho$ in the plane as:

$$
\rho(u, v)=\bar{\rho}(S(u, v)) \sqrt{\operatorname{det} M},
$$

where $\sqrt{\operatorname{det} M}$ accounts for the area change between $\mathcal{S}$ and $\mathcal{U}$. Similarly, a second-order tensor $\bar{\sigma}$ that acts on tangent vectors of the surface expressed in $\mathbb{R}^{3}$ defines a tensor $\sigma$ in the plane through:

$$
\sigma(u, v)=\nabla S(u, v)^{t} \bar{\sigma}(S(u, v)) \nabla S(u, v) .
$$

This setup will allow us to reexpress the $3 \mathrm{D}$ equilibrium conditions of masonry surface in 2D. We also refer to [Angelillo et al. 2012] for a rigorous derivation of this setup obtained by considering $3 \mathrm{D}$ equations in the asymptotic limit of singular statically admissible stress field concentrated on a surface lying inside the masonry structure.

Discrete Setup. For computational purposes, we will use a planar triangulation $\mathcal{T}$ discretizing the domain $\mathcal{U}$. We call $V$, $E$, and $F$ the set of vertices, edges, and triangles of the simplicial mesh, respectively. The positions of vertices are denoted as $\mathbf{u}_{i}=\left[u_{i}, v_{i}\right]$, and their associated heights are $z_{i}=z\left(u_{i}, v_{i}\right)$, while $|i j|$ indicates the length of edge $i j$ and $|i j k|$ indicates the area of triangle $i j k$ in 2D. Triangles are oriented counterclockwise, and edges are given an arbitrary (but fixed thereafter) orientation. We call $\mathcal{N}(i)$ the set of vertices that share an edge with $i$, and we use $\phi_{i}$ to denote the piecewise linear basis function over $\mathcal{T}$ for vertex $i$ with $\phi_{i}\left(\mathbf{u}_{j}\right)=\delta_{i j}$. Thus, the height field $z(\mathbf{u})=\sum_{i \in V} z_{i} \phi_{i}(\mathbf{u})$ represents a simplicial, piecewise-linear masonry structure. Finally, we denote by $\mathbf{d}_{0}$ the transpose of the incidence matrix of vertices and edges $(|E|$ rows, $|V|$ columns), where each row contains a single +1 and -1 for the endpoints of a given edge (the sign being determined from the chosen edge orientation), and zero otherwise; and by $\mathbf{d}_{1}$ the transpose of the incidence matrix of edges and faces $(|F|$ rows, $|E|$ columns), with +1 or -1 entries according to the orientation of edges as one moves counterclockwise around a face.

\subsection{Continuum mechanics description of equilibrium}

A self-supporting surface $\mathcal{S}$ is in equilibrium if the compressive forces at play compensate for the dead load created by the surface mass density $\bar{\rho}$ in a gravitational field $\mathbf{g}=[0,0,-g]$. This amounts to a surface stress tensor $\bar{\sigma}$ defined by a symmetric, negative semidefinite matrix that satisfies:

$$
\nabla \cdot \bar{\sigma}=[0,0, \bar{\rho} g] .
$$

One can conveniently rewrite this equilibrium condition as two separate equations using the 2D scalar field $\rho$ and tensor $\sigma$ defined via Eqs. (2) and (3):

$$
\nabla \cdot \sigma=0 \quad \text { and } \quad \nabla \cdot(\sigma \nabla z)=\rho g .
$$

The first equation indicates that the stress tensor $\sigma$ on $\mathcal{U}$ is divergence-free (i.e., the divergence of each of the columns of $\sigma$ is zero); the second relation equates an elliptic operator on the height to the local dead load in order to enforce balance. This last equation can be further reduced to $\Delta^{\sigma} z=\rho g$, where $\Delta^{\sigma}$ is the Laplacian operator in the metric induced by the stress $\sigma$. Moreover, two-dimensional stress tensors over simply connected domains can be encoded simply as the Hessian of a real-valued function $\psi$ negated and rotated on both sides by $\pi / 2$ [Green and Zerna 2002]. Called the Airy stress function, this function must be convex to enforce the compressible nature of stress tensor (Fig. 1).

\subsection{Existing discrete approximations of equilibrium}

The aforementioned differential description of equilibrium can naturally be leveraged to develop numerical schemes for the generation of self-supporting masonry structures. Next we summarize previous approaches before introducing our rationale for simplicial masonry.
Finite/Discrete Elements. Discretized balance equations can be derived through finite element analysis for a finite-dimensional approximation of the surface-typically, a triangle or quad mesh. However, traditional displacement-based conforming and low-order finite element methods applied to this problem can lead to indeterminacy or divergence under refinement of the solution [Giaquinta and Giusti 1985]. Stress-based discrete element methods were introduced to remediate these issues, but they often required mesh elements to be aligned with the principal directions of stress (see, for instance, [O'Dwyer 1999]). This unsavory constraint was further removed through the use of non-conforming finite elements, where stress is expressed via a discretized Airy stress function [Fraternali et al. 2002; Fraternali 2010; Fraternali 2011; Angelillo et al. 2012]. Since the existence of a stress field deriving from a Hessian matrix of an Airy function is only guaranteed for simply-connected domains, this tight and efficient discrete formulation restricts the topology of the surface.

Force Diagrams. Another well-studied approach is to approximate the continuum medium with an equivalent truss structure: the edges of a mesh are seen as the support of a force diagram acting on the nodes, thereby approximating the stress field through uniaxial singular stresses. Such a thrust network analysis (TNA) [Block and Ochsendorf 2007; Block 2009] only requires a negative value $\sigma_{i j}$ per edge $e_{i j}$, interpreted as a force density, to effectively encode the stress tensor. The equilibrium equations for each interior vertex $\mathbf{u}_{i}$ with height $z_{i}$ are then reduced to:

$$
\left\{\begin{array}{l}
\sum_{j \in \mathcal{N}(i)} \sigma_{i j}\left(u_{j}-u_{i}\right)=0 \\
\sum_{j \in \mathcal{N}(i)} \sigma_{i j}\left(v_{j}-v_{i}\right)=0 \\
\sum_{j \in \mathcal{N}(i)} \sigma_{i j}\left(z_{j}-z_{i}\right)=m_{i} g
\end{array}\right.
$$

where $m_{i} g$ is the local (lumped) gravitational load on vertex $i$. Despite great practical relevance stemming from its elegant simplicity, there is no rigorous analysis on the accuracy and convergence of TNA. The recent work of [Vouga et al. 2012] proposed to help architects design self-supporting structures by applying the TNA model on polygonal meshes; they solved for edge values $\sigma_{i j}$ by iteratively minimizing the residual of the TNA equations in the least-squares sense while best matching a user-given height field. Unfortunately, this least-squares based methodology is not without shortcomings: for mixed boundary conditions on the height, the resulting system of equations is in general overconstrained, and it may thus fail to find a self-supporting solution without resorting to user interaction.

Towards a Hybrid Approach. It is worth noting that the modeling of a continuous membrane through a discrete force network [Block 2009] closely matches the non-conforming approximation of the equilibrium problem [Fraternali 2011]. In particular, they both point out the singular nature of the stress (which is "lumped" along mesh edges only) and the importance of a reciprocal diagram to the planar orthographic projection of the surface mesh. There are, however, a few significant differences. For instance, Block [2009] favors primal and dual graphs with corresponding edges intersecting at a fixed angle, but Fraternali [2011] uses a barycentric dual graph instead. This difference stems from the physical interpretation of the dual graph: while the latter only needs well-formed dual cells over which the stress can be averaged in order to weakly enforce equilibrium, the former associates a reciprocal diagram to the existence of edge-aligned forces that cancel out the gravitational loading on each vertex. Our approach, described next and based on numerical homogenization, can be seen as reconciling these two methods for simplicial masonry structures, as we will inherit the conceptual simplicity of TNA while still leveraging finite elements literature for rigorous convergence analysis and bounds on accuracy. In particular, we will introduce a discrete formulation for which both conditions in Eq. (5) will be precisely enforced. 


\subsection{Upscaling of Self-Supporting Surfaces}

We now introduce our finite-dimensional characterization of equilibrium for masonry structures. Derived from an edge-based discretization of symmetric tensors and leveraging a geometric interpretation of the diagram of forces at play, it will serve as the foundation of our computational approach to simplicial self-supporting form finding.

Masonry equilibrium as upscaled elliptic problem. In $\S 2.2$ we formulated the condition of equilibrium of masonry structures as an elliptic problem in the space of divergence-free, symmetric, negative, and semi-definite tensors (Eq. (5)). This exact case (up to sign) appeared in the very different context of electric impedance tomography with rough conductivity coefficients in [Desbrun et al. 2013], where a numerical homogenization (or upscaling) of the equations were proposed via a harmonic change of coordinates. In particular, it was pointed out that a symmetric tensor $\sigma$ in 2D can be conveniently, but rigorously discretized on a simplicial mesh $\mathcal{T}$ by a scalar value $\sigma_{i j}$ for every (unoriented) edge $i j$ using the piecewise linear basis functions $\phi_{i}$ and $\phi_{j}$ as:

$$
\sigma_{i j}=-\int_{\mathcal{U}} \nabla \phi_{i}{ }^{t} \sigma \nabla \phi_{j} d u d v .
$$

Properties of the continuous tensor $\sigma$ were shown to carry over as simple properties on the coefficients $\sigma_{i j}$. For instance, the negative semi-definiteness of the continuous tensor corresponds to negative values of $\sigma_{i j}$. Moreover, due to the tensor $\sigma$ being divergence-free, the authors proved that for every vertex $i$,

$$
\sum_{j \in \mathcal{N}(i)} \sigma_{i j}\left(u_{j}-u_{i}\right)=0, \quad \text { and } \sum_{j \in \mathcal{N}(i)} \sigma_{i j}\left(v_{j}-v_{i}\right)=0 .
$$

Finally, the elliptic operator in Eq. (5) was then expressed in weak form on $\mathcal{T}$ as $\sum \sigma_{i j}\left(z_{j}-z_{i}\right)$. Consequently, a direct application of their finite elements methodology to our masonry case recovers the TNA formulation of Eq. (6).

Connection to DEC. The use of edge values to encode the stress tensor fits well the formalism of discrete exterior calculus (DEC, see [Desbrun et al. 2007; Grady and Polimeni 2010]), which will allow us to use a more compact notation expressing both the continuous and discrete equations at play. Indeed, discretizing a symmetric tensor with a value per unoriented edge complements the discrete version of a differential form (i.e., an antisymmetric tensor), which uses a value per oriented edge instead. In fact, $\sigma_{i j}$ can be interpreted, up to the sign, as a discrete Hodge star deriving from the continuous metric $\sigma$. Notice that if $\sigma$ is minus the identity (i.e., the negated $\mathrm{Eu}$ clidean metric on $\mathcal{U}$ ), one finds the well-known cotangent-based diagonal Hodge star widely used in geometry processing. (See [Zayer et al. 2005] for a related expression in the context of quasi-conformal maps with piecewise-constant tensors.) More generally, the diagonal matrix $\star_{1}^{\sigma}=\left\{\star_{i j}^{\sigma}:=-\sigma_{i j}\right\}_{i j \in E}$ is the discrete Hodge star for one-forms associated to the metric corresponding to the planar stress tensor $\sigma$. Note that the compressive nature of the stress imposes that the discrete Hodge star values must all be positive. Moreover, Eqs. (7) resulting from the divergence-freeness of the stress tensor $\sigma$ can be concisely rewritten as

$$
\mathbf{d}_{1}^{t} \star_{1}^{\sigma} \mathbf{d}_{0} \mathbf{u}=0 .
$$

One can readily check this corresponds to a statement of linear precision for the $\sigma$-induced discrete Laplacian $\Delta^{\sigma}=\mathbf{d}_{1}^{t} \star_{1}^{\sigma} \mathbf{d}_{0}$ on $\mathcal{U}$, since this discretization of the elliptic operator $\nabla \cdot(\sigma \nabla)$ applied to the $u$ and $v$ coordinates of the mesh $\mathcal{T}$ returns zero.

Equilibrium equations. Finally, the equilibrium equations that the height $z$ must satisfy can be given in a weak form, by integrating the differential equation over each planar dual cell of $\mathcal{T}$ :

$$
\mathbf{d}_{1}^{t} \star_{1}^{\sigma} \mathbf{d}_{0} z=\star_{0} \rho g,
$$

where $\star_{0}=\left\{\star_{i}\right\}_{i \in V}$ is the conventional discrete Hodge star for zero-forms on $\mathcal{T}$ [Desbrun et al. 2007]. Note the presence of two different Hodge stars: while we discussed the emergence of a stressinduced Hodge star $\star_{1}^{\sigma}$ for discrete one-forms that provides a geometric realization of the force diagram, the second Hodge star $\star_{0}$ is for zero-forms and represents an integration over each (barycentric, circumcentric, or otherwise) partition of the mesh. This latter Hodge star corresponds to the common practice of "mass lumping" in computational mechanics. With Eqs. (8) and (9), we have a discrete formulation mirroring the continuous conditions of Eq. (5).

\subsection{Stress-induced orthogonal dual diagram}

The properties of the scalar values $\sigma_{i j}$ we listed above also have a simple geometric implication due to the Maxwell-Cremona theorem [Wardetzky et al. 2007]: it implies that any divergence-free discrete stress tensor is associated to an orthogonal (reciprocal) Poincare dual diagram to the simplicial planar mesh for which the supporting lines of primal and dual edges are perpendicular. Consequently, a simplicial surface is self-supporting if and only if exists a stress-induced orthogonal dual mesh, corresponding to the TNA force diagram in [Block and Ochsendorf 2007; Vouga et al. 2012]. Conversely, the requirement of a divergence-free stress tensor removes the need to consider non-orthogonal dual diagrams.

Reduced coordinates of orthogonal dual diagram. An orthogonal dual diagram to a given planar primal mesh can be defined by a set of dual vertex locations with added constraints to satisfy the orthogonality condition between primal and dual edges: this characterization was first used in the context of conformal parameterization [Mercat 2001] before being adopted for selfsupporting surface design through thrust network analysis [Block and Ochsendorf 2007; Vouga et al. 2012]. We instead introduce a set of reduced coordinates that encode all possible orthogonal dual diagrams for a primal mesh of arbitrary topology. Recall that orthogonal dual structure in a single triangle exists if and only if three perpendicular bisectors crossing the triangle edges have a common intersecting point. This condition, which must hold on every triangle of a given simplicial mesh, was explicitly written in [Glickenstein 2005] as:

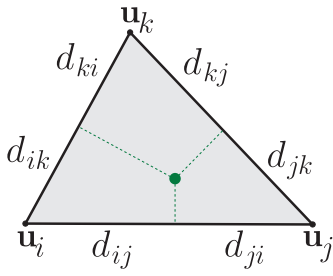

$$
\left(d_{j i}^{2}-d_{i j}^{2}\right)+\left(d_{k j}^{2}-d_{j k}^{2}\right)+\left(d_{i k}^{2}-d_{k i}^{2}\right)=0,
$$

where $d_{a b}$ is the signed distance between the vertex $\mathbf{u}_{a}$ and the intersection of the lines supporting edge $a b$ and its perpendicular bisector (note that $d_{a b}+d_{b a}=|a b|$; see inset). We can rewrite this condition in the language of algebraic topology (using DEC) so as to uncover a parametrization of the whole space of orthogonal dual diagrams for a given triangulation. Indeed, define a primal discrete one-form $\omega$ with value $\omega_{i j}=d_{j i}^{2}-d_{i j}^{2}$ per oriented edge $i j$. Then Eq. (10) states that an orthogonal dual mesh is fully defined by a closed primal one-form $\omega$, i.e., $\mathbf{d}_{1} \omega=0$ : once such a closed form $\omega_{i j}$ is known, $d_{i j}$ can be deduced from the given primal lengths $|i j|$ (Eq. (12)). Furthermore, Hodge decomposition (see, e.g., [Desbrun et al. 2007]) can be invoked to rewrite this closed one-form as a function of a primal zero-form $w$ and a (non-integrable) primal harmonic one-form $\eta$, i.e.,

$$
\omega=\mathbf{d}_{0} w+\eta
$$

with normal and tangential boundary conditions for the two terms respectively. (Intuitively, the one-form $\eta$ corresponds to a harmonic vector field which cannot be written as the gradient of a scalar function, hence the term non-integrable.) Finally, recall that harmonic one-forms admit a small set of $\beta_{1}$ basis one-forms for an arbitrary bounded planar triangulation, where $\beta_{1}$ is the first Betti number indicating the number of holes in the domain (see a simple computational procedure to compute this basis in, for instance, [Tong et al. 
2006]). We can thus compactly encode the one-form $\eta$ at an edge $i j$ as a linear combination of these basis one-forms, i.e.,

$$
\eta_{i j}=\sum_{q=1 . . \beta_{1}} c_{q} \gamma_{i j}^{q}
$$

where $\gamma^{q}$ is a basis element for harmonic one-forms (with unit circulation around its associated hole) and $c_{q}$ is a real-valued holeindexed coefficient. An orthogonal dual mesh can thus be arbitrary constructed by assigning a value $w_{i}$ per vertex (zero-form) and a set of $\beta_{1}$ values $\left\{c_{q}\right\}_{q=1 . . \beta_{1}}$ defining a harmonic one-form. Note that the use of $\mathbf{d}_{0}$ makes the zero-form defined only up to a constant. The dimensionality of the full space of orthogonal dual meshes for a given mesh $\mathcal{T}$ discretizing a domain $\mathcal{U}$ is therefore $(|V|-1)+\beta_{1}$, i.e., the number of vertices of $\mathcal{T}$ (minus one to remove the additive constant of the zero-form) plus the number of holes $\beta_{1}$ of the domain $\mathcal{U}$. An example of how the dual diagram is changed by hole-indexed coefficients is in Fig. 2; notice the concentration of stress near the hole, linked to the Saint-Venant principle of structural engineering. Even though we will only consider planar meshes in this work, this result is also valid for manifold triangulations of arbitrary genus.

Closed-form expressions of edge stress values. Another consequence of the Maxwell-Cremona theorem is that the values $\sigma_{i j}$ (or, equivalently, the discrete Hodge star for one-forms $\star_{1}^{\sigma}$ ) are directly linked to the geometry of the primal-dual structure: each edge value $\sigma_{i j}$ is (minus) the ratio of dual length to primal length of edge $i j$ - fitting the definition of the diagonal Hodge star on circumcentric duals [Desbrun et al. 2007] and on weighted triangulations [Glickenstein 2005; Mullen et al. 2011]. We can then provide closed-form expression of the edge values corresponding to a divergence-free tensor $\sigma$. Denote by $\mathbf{c}_{i j k}$ the position of the stress-induced dual node to triangle $i j k$, and by $\mathbf{c}_{i j}$ the position of the intersection of the supporting lines of the primal edge $i j$ and of its stress-induced dual (see inset). Additionally, define $h_{j k i}$ as the signed distance between $\mathbf{c}_{i j k}$ and $\mathbf{c}_{i j}$, with a positive sign if the triangle $\left(\mathbf{c}_{i j k}, \mathbf{u}_{i}, \mathbf{u}_{j}\right)$ has the same orientation as the triangle $\left(\mathbf{u}_{i}, \mathbf{u}_{j}, \mathbf{u}_{k}\right)$,

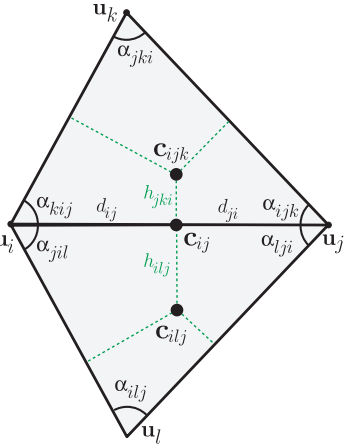
and negative otherwise. One finds, for triangle $i j k$, the following expressions:

$$
\begin{gathered}
d_{i j}=\frac{|i j|^{2}+\omega_{j i}}{2|i j|}, \quad d_{j i}=\frac{|i j|^{2}+\omega_{i j}}{2|i j|}, \\
h_{j k i}=\frac{|i j| \cot \alpha_{j k i}}{2}+\frac{\cot \alpha_{k i j}}{2|i j|} \omega_{k j}+\frac{\cot \alpha_{i j k}}{2|i j|} \omega_{k i} .
\end{gathered}
$$

Then, from these functions of $\omega_{i j}$, the stress-induced Hodge star value $\star_{i j}^{\sigma}$ is expressed as the ratio of the dual length (denoted $h_{i j}$ ) to the primal length of the stress-induced diagram edge $i j$, i.e.,

$$
\star_{i j}^{\sigma}=\frac{h_{i j}}{|i j|}, \quad \text { with } h_{i j}=h_{j k i}+h_{i l j} .
$$

Notice from this last formula that the $\sigma$-induced Hodge star has only positive coefficient (corresponding to pure compression) if and only if every dual edge has a positive length, i.e., if the primal mesh $\mathcal{T}$ is a regular triangulation [Preparata and Shamos 1985].

\subsection{Boundary conditions}

Proper handling of the boundaries is crucial to both the numerical treatment (i.e., to avoid overconstrained equations) and the quality of the results (i.e., to guarantee self-support). Our discrete setup leads to simple and flexible boundary conditions.
Stress on boundaries. First recall that since only compressive forces should be at play, the $\sigma_{i j}$ edge values must all be negative; the boundary $\sigma_{i j}$ values are no exception, and they define boundary dual edges on the stress-induced dual diagram. Moreover, since boundary vertices may be fixed (via a buttress or other construction artifacts), enforcing a divergence-free stress tensor at the boundary is necessary only for non-fixed vertices. In our setup, the divergence-free condition at the boundary is enforced in weak form by integrating the strong form over the planar region $V_{i}$ formed by the circumcentric dual cell associated to $i$ clamped at the boundary $\partial \mathcal{T}$; it thus reads:

$$
0=\int_{V_{i}} \nabla \cdot \sigma=\int_{\partial V_{i}} \sigma \mathbf{n}=\sum_{j \in \mathcal{N}(i)} \star_{i j}^{\sigma}\left(\mathbf{u}_{i}-\mathbf{u}_{j}\right)+\int_{V_{i} \cap \partial \mathcal{T}} \sigma \mathbf{n} .
$$

The first term of this equation matches the definition of divergencefreeness for interior points (Eq. (8)), yet with an important difference: boundary edges have values $h_{i j}$ made out of only a partial dual edge (one of the two terms in Eq. (14) is zero since there is only one triangle adjacent to $i j$ ). We thus complete these dual edges by adding boundary dual lengths $h_{\infty}=\left\{h_{i \infty j}\right\}_{i j \in \partial \mathcal{T}}$ : with these additional boundary variables, the resulting stressinduced Hodge star values $\star_{1}^{\sigma}$ (that must be positive to enforce compression) are well defined even at the boundary as dual/primal edge length ratios. Notice that the resulting stress-induced dual boundary edges represent the (rotated) tangential boundary forces (just like the internal dual edges represents the rotated edge-aligned forces at play in the domain). The compressive normal forces on the boundary are instead reflected in the second term of the equation above: if we define the (negated) normal stress at the boundary edge $i j$

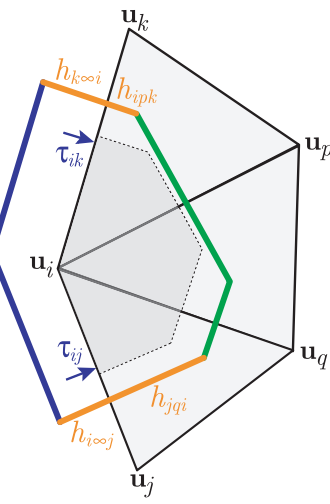
as $\tau_{i j} \geq 0$, the resulting normal force on $i j$ is thus equal to $-\tau_{i j} \mathbf{n}_{i j}$ with $\mathbf{n}_{i j}$ being the length-weighted outward normal of $i j$. The integration of the normal forces along $V_{i} \cap \partial \mathcal{T}$ with adjacent boundary vertices $j$ and $k$ (see inset) becomes ${ }^{1}$ :

$$
-\frac{1}{2} \tau_{i j} \mathbf{n}_{i j}-\frac{1}{2} \tau_{k i} \mathbf{n}_{k i}
$$

These tangential and normal boundary terms offer a discrete analog to the continuous, general stress boundary condition on $\sigma \mathbf{n}$ [Giaquinta and Giusti 1985], and brings a more flexible and general boundary handling in the design of self-supporting surfaces.

Boundary equilibrium. Consider now the balance equations, which this time involve heights $z_{i}$ at the boundary. There are only two types of boundary condition for a boundary vertex:

- if a boundary vertex is anchored, its height $z$ is fixed (generally to zero if the height is supposed to be on the ground), and no balance equation is needed for this vertex;

- if a boundary vertex is not anchored (i.e., free), the balance equation is then activated.

The physical and geometric justifications of these two cases are straightforward. Indeed, the equilibrium equation for the height at a boundary vertex $i$ is written in weak form as before:

$$
\int_{V_{i}} \Delta^{\sigma} z=\int_{\partial V_{i}} \mathbf{n}^{t} \sigma \nabla z=\sum_{j \in \mathcal{N}(i)} \star_{i j}^{\sigma}\left(z_{i}-z_{j}\right)+\int_{V_{i} \cap \partial \mathcal{T}} \mathbf{n}^{t} \sigma \nabla z .
$$

Besides the $\star_{i j}^{\sigma}\left(z_{i}-z_{j}\right)$ terms which are also present for interior points, an additional integral of the boundary stress appears relating

\footnotetext{
${ }^{1}$ Note that the two $1 / 2$ coefficients come from our specific choice of $V_{i}$ as circumcentric dual cells, which split every primal edge into two equal parts; other cell decompositions would lead to different coefficients.
} 
$\nabla z$ to the boundary values $h_{\infty}$ and $\tau$. As in the divergence-freeness case, the values $h_{\infty}$ are incorporated in the dual lengths, while the normal component along the boundary segment $i j \cap V_{i}$ adjacent to the triangle $i j k$ is discretized following [Fisher et al. 2007] as:

$$
\frac{1}{2} \tau_{i j}\left(\cot \alpha_{i j k}\left(z_{i}-z_{k}\right)+\cot \alpha_{k i j}\left(z_{j}-z_{k}\right)\right) \text {. }
$$

Engineering considerations. Note that engineering design of masonry-like edifices needs a full control of the stresses at play in the structure. So while we proposed to simply skip the divergencefreeness and equilibrium equations for anchored boundary vertices, one may also adopt a more thorough description of the stress field by adding these equations back, and including the respective boundary compressions as variables for each vertex. Then one can control these values to either make sure they do not exceed a critical threshold that the anchor could not bear, or simply to make sure that these forces are also sufficiently compressive as a margin of safety. Similarly, hole-indexed coefficients $c$ and free boundary stresses $\tau$ can be either fixed based on engineering constraints such as prestressing, or simply optimized along with the other variables. While we do not explore all these specific engineering requirements, our formulation accommodates them naturally.

Special cases. Finally, we point out that our approach reproduces as special cases the boundary treatments presented in previous work. Employing Dirichlet or Neumann boundary conditions on the Airy function as in [Fraternali 2010; Angelillo et al. 2012] (enforcing both is known to be overconstrained) corresponds to enforcing the same conditions on the zero-form values $w_{i}$ at the boundary. Instead, free boundary dual edges intersecting in a single point as in [Block 2009; Vouga et al. 2012] corresponds to setting normal stresses $\tau$ to zero (Figs. 3 and 9). Note that the latter boundary condition pushes the extra boundary term in the equilibrium equation to zero as well. While these choices are valid, they only correspond to a subset of all possible boundary conditions.

\subsection{Discussion}

Our discretization provides a formal backdrop to the equations used in thrust network analysis [Block and Ochsendorf 2007], and inherits

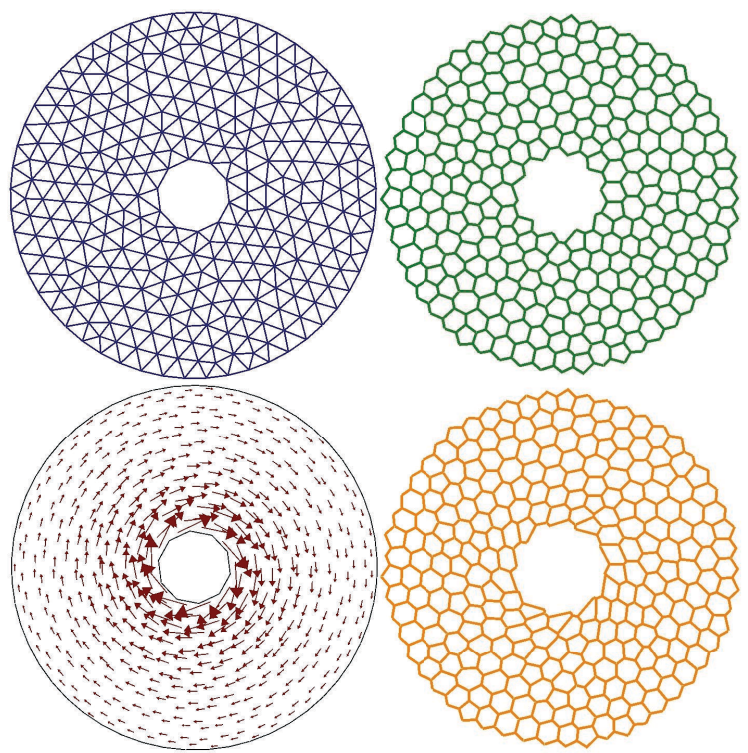

Figure 2: Effect of harmonic one-forms. If a harmonic one-form $\eta$ is added to a planar triangulation with non-trivial genus (top left), the stress-induced dual diagram (top right) is displaced (bottom right) by a non-integrable vector field (bottom left), adding a valuable degree of freedom to find selfsupporting shapes. Notice that the displacement is larger around the hole, and decreases rapidly due its harmonic nature. the convergence and accuracy analysis tools available in the finite elements literature [Desbrun et al. 2013]. Moreover, we managed to completely characterize the space of valid discrete equilibrium solutions, which will allow us to solve for self-supporting structures using the reduced set of variables $w$ and $c$ that fully describe the set of valid discrete divergence-free symmetric tensors $\sigma$. Many remarks are in order, as our results relate to previous work not only in masonry design, but also in computational geometry.

Weighted vs. regular triangulations. We parameterized the set of all orthogonal dual of a simplicial mesh with a primal zero-form and a harmonic one-form. Note that if the mesh $\mathcal{T}$ is simply connected, there exists no harmonic one-forms, so only vertex values are needed to span the space of orthogonal dual diagrams for a simply connected mesh. This special topology case was in fact explicitly stated by Glickenstein [2005], with the zero-form $w$ being referred to as vertex weights. (The reader can easily check that the expressions given in his paper match ours for the restrictive case $\omega=\mathbf{d}_{0} w$.) The resulting "weighted triangulations" were also shown useful for geometry processing [Mullen et al. 2011]. However, the case of an arbitrary domain topology was left unattended, probably due the fact that meshes in computational geometry are often studied as projections of higher-dimensional convex polytopes - which forbids the presence of holes. It should be noticed that, as a consequence, weighted Delaunay triangulations and regular triangulations are often assumed to be equivalent, but this statement ignores the $\beta_{1}$ additional dimensions available for the latter due to topology (Fig. 2).

Airy function. Although our formulation drastically differs from Fraternali's work, a formal connection to the Airy stress function is easily made, once again for the special case of simply connected domains. The Airy function comes from the integrability of the stress tensor seen as the (rotated and negated) Hessian of a scalar function [Green and Zerna 2002]. In the discrete setting, this function corresponds to the integrable part of the orthogonal dual mesh associated to the discrete stress values $\sigma_{i j}$ and thus it is defined through the zero-form $w$ via $\psi(\mathbf{u})=\frac{1}{2} \sum_{i}\left(\left\|\mathbf{u}_{i}\right\|^{2}-w_{i}\right) \phi_{i}(\mathbf{u})$. Geometrically, the Airy function can be seen as the lifting of the 2D mesh $\mathcal{T}$ to a paraboloid of height $\psi(\mathbf{u})$ as used in [Desbrun et al. 2013]; see Fig. 1. This geometric picture was previously used to construct power diagrams [Aurenhammer et al. 1998] and is related to the convex potential function found in optimal transport [Mérigot 2011]. Hence, we have formally established that the discrete Airy values $\psi_{i}$ in Fraternali's work are related to weights of the resulting weighted Delaunay triangulation through: $\psi_{i}=\frac{1}{2}\left\|\mathbf{u}_{i}\right\|^{2}-\frac{1}{2} w_{i}$. Besides extending his approach to arbitrary topology (i.e., non-zero $\beta_{1}$ ), our analysis offers a valuable discrete notion of divergence-freeness that his work did not exploit, and links the convex hull procedure he advocated to the construction of a weighted Delaunay triangulation.

Generalized Airy function. In the broader context of elastostatics, Fosdick and Schuler [2003] introduced a continuous generalization of the Airy stress function to domains with holes by incorporating the symmetric part of the derivative of smooth vector fields. Remarkably, our reduced set of coordinates for orthogonal dual diagrams provides a principled and complete discretization of such generalized representation of stress in the case of divergence-free tensors on simplicial meshes of arbitrary topology.

Lumped mass matrix. Finally, we point out that the specific choice of lumped mass matrix (in our case, $\star_{0}$ ) varies across the literature. While Fraternali [2011] uses the integral of the density over each barycentric dual cell of $\mathcal{T}$, Vouga et al. [2012] integrates over circumcentric dual cells. We also adopt circumcentric dual cells for simplicity, but alternatives are easy to incorporate. One may even consider using the space of all possible partitions of the domain as yet another set of degrees of freedom. Besides the changes in local dead load that other choices of dual cells generate, the only other 


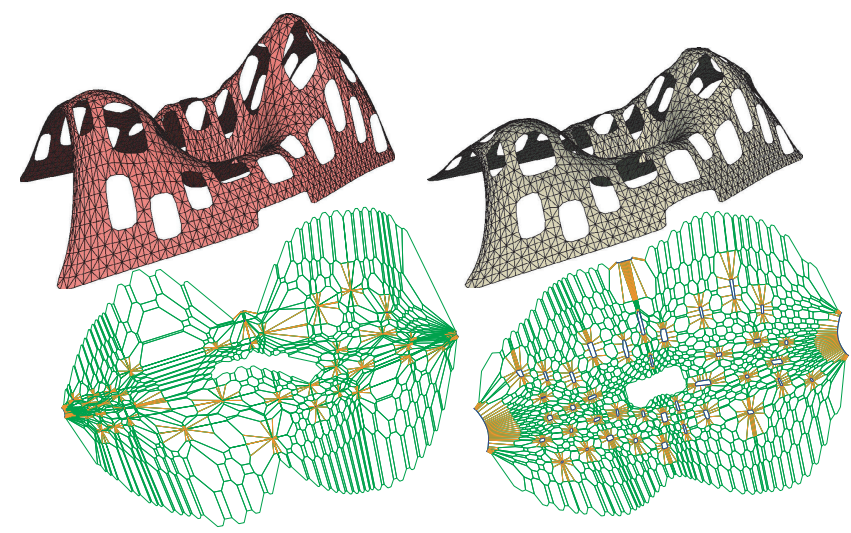

Figure 3: Boundary conditions. The normal stress imposed around holes can significantly affect the shape of a masonry structure; here, a shape from [Vouga et al. 2012] with several holes, where the boundary normal stresses are either set to zero (left; notice that the boundary (orange) dual edges meet at a point) or not (right; the normal forces are in dark blue).

difference that one needs to address is the treatment of boundary conditions: using two sub-variables $\tau_{i j}$ and $\tau_{j i}$ per boundary edge $i j$ representing the integration of the normal stresses on $V_{i} \cup i j$ and $V_{j} \cup j i$ respectively allows for a more general setup with minimal code modification.

\section{Variational Formulation}

Variational formulations for equilibrium equations have a long history in mechanics, and the specific case of masonry structures is no exception [Giaquinta and Giusti 1985; Fraternali et al. 2002; Fosdick and Schuler 2003; Fraternali 2011]. Our setup involving a primal simplicial mesh and a stress-induced orthogonal dual diagram turns out to also offer a convenient variational formulation, but now at the discrete level, which can then be used for computational purposes.

Equilibrium functional. We introduce an energy $\mathcal{E}$ that is a function of both the zero-form ("weights") $w=\left\{w_{i}\right\}_{i \in V}$ and the harmonic-form coefficients $c=\left\{c_{q}\right\}_{q=1 . . \beta_{1}}$ as follows:

$$
\mathcal{E}(w, c)=\sum_{i} z_{i}\left[\frac{1}{2} \sum_{j \in \mathcal{N}(i)} d_{i j} h_{i j}\right]-\sum_{i} w_{i}\left(\star_{i} \rho_{i} g\right) \text {. }
$$

Based on Eqs. (12) and (13), we note that this functional is quadratic in both variables $w$ and $c$. We also point out that the term in brackets is an analytical expression of the area of the stress-induced dual cell for vertex $i$, while the second term depends on the vertex load $\star_{i} \rho_{i} g$. To account for the boundary equilibrium conditions discussed in $\S 2.6$, we also incorporate to our energy $\mathcal{E}$ an extra term for every free boundary edge $i j$ :

$$
\begin{aligned}
& h_{i \infty j}\left(d_{i j} z_{i}+d_{j i} z_{j}\right) \\
& +\frac{1}{2} \tau_{i j}\left(w_{i}+w_{j}\right)\left(\cot \alpha_{i j k}\left(z_{i}-z_{k}\right)+\cot \alpha_{k i j}\left(z_{j}-z_{k}\right)\right) .
\end{aligned}
$$

Extremization conditions. Using the derivatives of the terms $d_{i j}$ and $h_{j k i}$ provided in the appendix, one finds that the gradient of $\mathcal{E}$ with respect to the weight $w_{i}$ of an interior vertex $i$ is:

$$
\nabla_{w_{i}} \mathcal{E}=\sum_{j \in \mathcal{N}(i)} \star_{i j}^{\sigma}\left(z_{i}-z_{j}\right)-\left(\star_{i} \rho_{i} g\right)
$$

We immediately deduce that a critical point for weights of $\mathcal{E}$ enforces exactly the balance equations on all interior vertices, and similarly for free boundary vertices if we include the additional boundary terms. Therefore, finding a self-supporting structure for a fixed set of heights, boundary anchors, and harmonic-form coefficients $c$ amounts to extremizing $\mathcal{E}$ subject to the linear inequality constraints $\star^{\sigma}, \tau \geq 0$ that enforce pure compression throughout the structure, plus the divergence-free condition at the free boundary vertices.

\section{Computational Form Finding Algorithms}

With a clear understanding of the geometry of the problem and of the variables at play, we can now provide an approach to the generation of self-supporting simplicial structures. We assume that an initial pointset $\left(\mathbf{u}_{i}, z_{i}\right)$ is given, as well as a connectivity that defines the planar mesh $\mathcal{T}$ with a non-overlapping orthographic projection, and with tags on boundary vertices indicating whether an anchored or free boundary condition is desired. In case the surface can not stand by itself with the given inputs, we need to (minimally) alter its shape to make it self-supporting. We give pseudocode of our overall form finding procedure in Fig. 4, and go through the numerical details of each step. We then discuss possible variants and design tools derived from this basic computational approach.

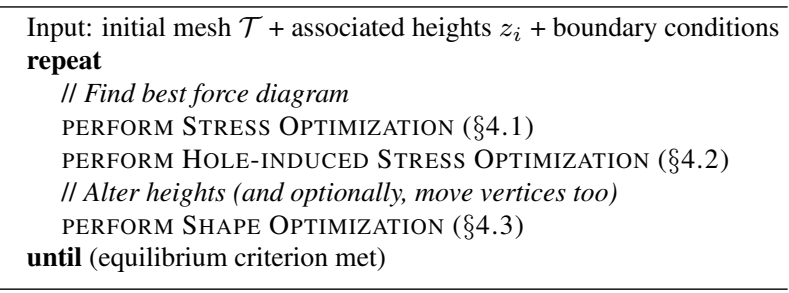

Figure 4: Pseudocode of our form-finding solver.

\subsection{Stress optimization}

We designed in $\S 3$ a variational principle whose gradient with respect to weights measures how self-supporting a structure is: if we are at a critical point (gradient equals zero), the structure is at equilibrium. Unfortunately, this energy is not necessarily convex depending on the input mesh; furthermore, there may not even be weights that enforce equilibrium for a given arbitrary shape while satisfying the inequality constraints. However, we previously noted that this energy is quadratic in the weights. So from our variational principle, we can find vertex weights and boundary values that best enforce equilibrium of non-anchored vertices in the $L_{2}$ sense by solving for:

$$
\operatorname{argmin}_{w, h_{\infty}, \tau}\left\|\nabla_{w} \mathcal{E}\right\|_{2}^{2} \text { s.t. } \star^{\sigma}, \tau \geq 0,(\nabla \cdot \sigma=0)_{\text {boundary }} .
$$

Note that the divergence-free constraint is necessary only for free boundary vertices due to the extra boundary terms described in $\S 2.6$. This quadratic energy with linear constraints will reach zero iff there exists a set of weights and boundary values defining a valid stress-induced orthogonal dual for which the heights $z_{i}$ satisfies the equilibrium equations. In the likely case there are no such weights (as the user may start from a very bad configuration), we find the weights which make the structure as close to equilibrium.

Our approach is similar in spirit to [Vouga et al. 2012], but we now compute the best stress configuration using a smaller set of equations. In fact, working in reduced coordinates enforces the divergence-free equations (Eq. (8)) exactly for all interior verticesthus we only have to solve for the remaining equilibrium equations (Eq.(9)), corresponding to two third less equations. As the size of the system is considerably reduced, solving this optimization to convergence is much more efficient: even on relatively small meshes, we get a $5 x$ speed up. Our method also resembles the work of [Block and Lachauer 2011]; however, while the latter computes a space of reduced coordinates through Gauss elimination of the TNA equations (Eq. (6)), which has cubic complexity in the mesh size, our approach provides the reduced coordinates in closed form (Eqs. (12) and (13)). More importantly, our boundary treatment in $\$ 2.6$ is more general and turns previous approaches such as [Block and Lachauer 2011; Vouga et al. 2012] from a potentially overconstrained problem to a systematically underconstrained problem. 

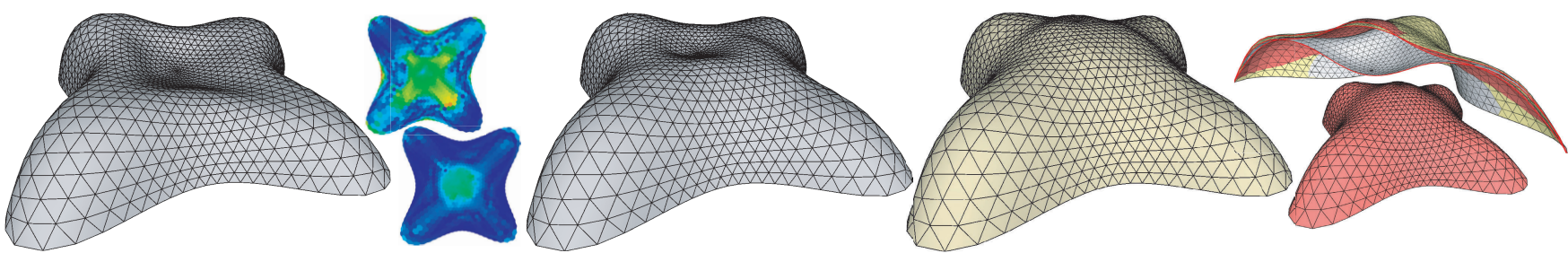

Figure 5: Height optimization. An input shape from [Vouga et al. 2012] is optimized, improving the residual of the equilibirum equations along the way (colormapped insets). Our form finding procedure removes the aphysical, concave center part of the shape. Compared to Vouga et al.'s result (red), we find a self-supporting configuration with a normalized Hausdorff and a $L_{2}$ distance to the initial shape of 0.04975 and 0.00758 respectively, while theirs are 0.04996 and 0.00831: their result suffers "sagging" of the initial shape near the anchored boundary, with less steep walls (see cut for comparisons).

\subsection{Hole-induced stress optimization}

Once the vertex weights and boundary values have been optimized, we can then further improve balance (if it is not already enforced) by optimizing for the harmonic-form coefficients $c$. This, of course, is only performed if the domain has at least one hole $\left(\beta_{1}>0\right)$, and we proceed as in the previous case by performing a solve for:

$$
\operatorname{argmin}_{c, h_{\infty}, \tau}\left\|\nabla_{w} \mathcal{E}\right\|_{2}^{2} \text { s.t. } \star^{\sigma}, \tau \geq 0,(\nabla \cdot \sigma=0)_{\text {boundary }} .
$$

Because the above energy is also quadratic in the coefficients $c$, this optimization is particularly simple: we have a quadratic form of only $\beta_{1}$ coefficients to minimize under constraints. This allows us to further adjust the stress-induced dual diagram in order to improve balance, a step ignored by all previous approaches. See a few results of stress and hole-induced stress optimization on various input meshes in Figs. 3, 8 and 9.

\subsection{Shape optimization}

Finally, assuming that optimizing weights, boundary values, and harmonic components have not yielded equilibrium, we must modify the assigned height values $z_{i}$ to find a self-supporting surface. This shape optimization step can take on various forms. Vouga et al. [2012] advocated a change of heights $\left\{z_{i}\right\}$ in concert with a change of positions $\left\{\mathbf{u}_{i}\right\}$ to reach a $L_{2}$ minimum of the equilibrium conditions. A change in node positions was beneficial in their case because it helped enforce the divergence-free condition which, unlike in our approach, was not satisfied by default. This particular approach has the inconvenience of not separating shape control and mesh quality, which can change the initial shape quite significantly in the process, as demonstrated in Fig. 5. Methods enforcing the divergence-freeness through the Airy stress function (for simply connected domains) also proposed shape optimization of various forms. For instance, Fraternali [2011] was removing equilibriumviolating vertices altogether until an equilibrium was reached, but this form finding approach may destroy the mesh quality. Instead, Angelillo et al. [2012] proposed to freeze the parts of the surface already satisfying equilibrium and solve for the optimal remaining heights. However, this binary update reduces the smoothness of the results, and ends up taking time to converge.

Here again, we leverage our variational approach to provide a simple and robust numerical approach for smoothly modifying the shape to become self-supported while staying as faithful as possible to the original input shape. To achieve this effect, we use a minimization with soft constraints: we minimize with respect to each $z_{i}$ the norm of the residual of the balance equations, where each vertex $i$ is weighted inversely proportional to its local balance residual. In other words, we optimize the heights by giving more leeway to vertices that are significantly violating the equilibrium condition, while vertices already near equilibrium will keep their heights almost unchanged. This weighted- $L_{2}$ residual minimization has the advantage of only affecting the shape in parts that are significantly not self-supporting: Fig. 5 shows that while concave parts of the original shape are necessarily altered to reach self-support, the rest of the surface is mostly unaffected. Other weighting strategies can of course be designed based on user preference.

As part of the shape optimization procedure, we also introduce an optional step of mesh smoothing: based on the current vertex positions $\mathbf{u}_{i}$ in the plane, we compute an update of the Optimal Delaunay Triangulation optimization introduced in [Alliez et al. 2005]: we move the coordinates of the mesh $\mathcal{T}$ such that the mesh elements on the surface are more equilateral. Note that this may actually reduce the quality of the shape of triangles in the plane, but the simplicial masonry structure will be better geometrically discretized. This optional step serves several purposes: first, it guarantees that our algorithm does not create degenerate elements; it also helps getting smoother resulting shape since the sampling quality is improved; finally, it favors the creation of concavities on free boundaries.

\subsection{Variants}

Many possible variants of our algorithm can be implemented. In particular, margin of errors are important in engineering to allow for small construction errors. One can modify the solvers to enforce a number of physical properties. For instance, the constraint that each $\sigma_{i j}$ be zero or negative can be changed to be bounded away from zero (to enforce non-negligible, but material-adequate compression), which we accommodate with ease by changing the constraints in our optimization. Similarly, the boundary forces can be assigned or optimized based on engineering needs. One could enforce constraints on the force diagram as well, by either bounding the maximum dual edge lengths or penalizing wild variations in dual lengths. We could also prescribe the normal stress for free boundary vertices, to deal with the case where a hole is attached to, say, a pole, in order to suspend the whole masonry structure. By setting the normal stress terms to zero, we can further enforce the boundary dual edges to intersect in a single point, as done in [Block 2009; Vouga et al. 2012] (Figs. 3 and 9). A user-defined varying mass density can also be defined if the masonry structure is supposed to withstand an extra load (Fig. 6). Finally, we point out that we assumed a given connectivity in our approach. However, we may

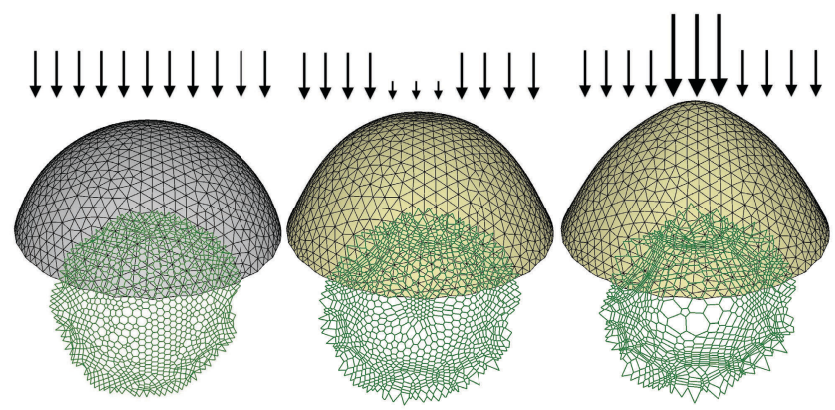

Figure 6: Load bearing. By changing the load in a small region at the top of an initial half-sphere shape, various dome shapes can be generated. In order: constant mass, lighter top $(\times 0.1)$, and heavy top $(\times 5)$. 
also alter the connectivity either based on user-guidance, or during the form finding procedure to optimize for, e.g., maximum stress. Adaptive meshing may also be an interesting avenue to explore to refine the shape where stresses are high. In all cases, our knowledge of the reduced coordinates for which a structure is self-supporting leads to more compact equations and faster solves.

\subsection{Timing and accuracy}

We used CGAL [2012] as our mesh library, and IPOPT [Wächter and Biegler 2006] as our numerical optimization library. Equilibrium of all our examples was enforced by ensuring that the $L_{\infty}$ norm of the gradient of the residual of non-anchored vertices is below 1e- 6 , which took between 5 and 20 iterations of our form-finding iterative solver depending on the input model size. Typical timings for the various form finding tests we made were at most two minutes. We also compared in Fig. 7 our stress optimization timing to Vouga et al. [2012] and found a systematic improvement varying from a factor 2 to 7 for meshes of various sizes.

\begin{tabular}{|l|c|c|c|}
\hline Model & $\mathrm{V} ; \mathrm{E} ; \mathrm{F}$ & Vouga et al.'s & Ours \\
\hline Cheese-model (Fig. 3) & $2348 ; 6218 ; 3832$ & 13.078 & 9.8 \\
Lilium (Fig. 5) & $1201 ; 3504 ; 2304$ & 4.136 & 0.887 \\
Variable load (Fig. 6) & $1156 ; 3368 ; 2213$ & 3.65 & 0.77 \\
Shifted barrel vault (Fig. 8) & $310 ; 836 ; 527$ & 0.24 & 0.15 \\
Moebius igloo (Fig. 8) & $702 ; 2006 ; 1304$ & 1.3 & 0.4 \\
Dome with hole (Fig. 8) & $1656 ; 4842 ; 3186$ & 7.7 & 1.5 \\
Groin vault (Fig. 8) & $2943 ; 8569 ; 5627$ & 23.6 & 4.1 \\
Free vault (Fig. 9) & $360 ; 1000 ; 640$ & 0.387 & 0.18 \\
Dome with doors (Fig. 9) & $577 ; 1600 ; 1024$ & 0.877 & 0.368 \\
Video-surface (Fig. 9) & $1131 ; 3194 ; 2064$ & 3.55 & 0.98 \\
Cas-model (Fig. 9) & $5951 ; 17472 ; 11520$ & 90.1 & 18.0 \\
Oval dome (no figure) & $12250 ; 36500 ; 24251$ & 443.467 & 61.277 \\
\hline
\end{tabular}

Figure 7: Timing. Comparisons between dual optimization timings (in seconds) from Vouga et al. [2012] and our approach. All results were clocked on an Intel Core i7 $2.2 \mathrm{GHz}$ laptop with 4GB RAM.

\section{Conclusions}

Our work on simplicial masonry provides a discrete theory of equilibrium for purely compressive structures that can support their own weight. We showed that the well-known continuous equations from the rich mechanical engineering literature find simple, discrete

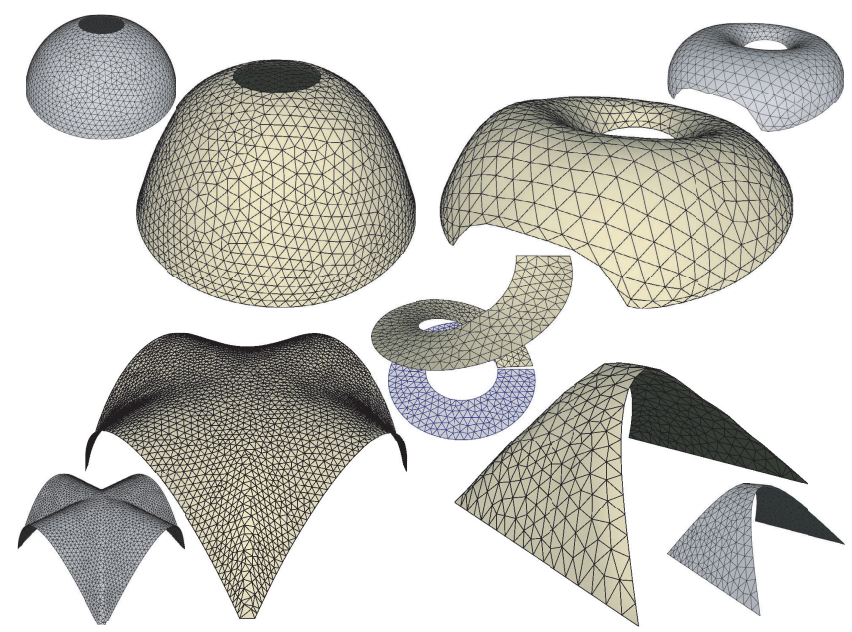

Figure 8: Gallery. Free-standing shapes can be obtained with our approach. While the Moebius igloo was our design (top right), the dome with a circular oculus (top left, similar to Rome's Pantheon; notice how the opening dilates during optimization to enforce equilibrium), the groin vault (bottom left), the shifted barrel vault (bottom right), and the spiral staircase (center) are all classic masonry structures (insets show the initial meshes we used). equivalents. We also exploited these properties to formulate a set of reduced coordinates in order to encode equilibrium and boundary conditions. Finally, we leveraged this tight formulation to produce a computational form finding procedure to alter a reference shape into a free standing simplicial masonry structure.

As future work, our discretization could be used to predict or simulate the development of cracks over time based on the stress field's principle directions as postulated in [Fraternali 2011]. It may also be interesting to apply our setup to the "opposite" case of wrinkled membranes, for which the forces at play are tensile instead of compressive [Wong and Pellegrino 2006]. Many of the properties we mention should remain valid as is, with an opposite sign. Generalization to arbitrary structures, including reinforced concrete, may also bring a complementary set of computational techniques to the traditional finite elements tools currently used in engineering firms.

Acknowledgements. Thanks to Etienne Vouga for early discussions and data, David Bommes for his IPOPT wrapper, and Keenan Crane for making the igloo model. Partial funding was provided by NSF grant CCF-1011944, ERC Starting Grant "Robust Geometry Processing" (257474), and gifts from Pixar Animations Studios and Walt Disney Animation Studios.

\section{References}

Alliez, P., Cohen-Steiner, D., Yvinec, M., And Desbrun, M. 2005. Variational tetrahedral meshing. ACM Trans. Graph. 24, 3 (July), 617-625.

Angelillo, M., Babilio, E., And Fortunato, A. 2012. Singular stress fields for masonry-like vaults. Continuum Mechanics and Thermodynamics, 1-19.

Aurenhammer, F., Hoffmann, F., And Aronov, B. 1998. Minkowski-type theorems and least-squares clustering. Algorithmica 20, 1, 61-76.

Block, P., AND LACHAUER, L. 2011. Closest-fit, compressiononly solutions for free form shells. In IABSE/IASS London Symposium, Int. Assoc. Shell Spatial Structures.

BLOCK, P., AND OCHSENDORF, J. 2007. Thrust network analysis: A new methodology for three-dimensional equilibrium. J. Int. Assoc. Shell and Spatial Structures 48, 3, 167-173.

Block, P. 2009. Thrust Network Analysis: Exploring Threedimensional Equilibrium. PhD thesis, Department of Architecture, Massachusetts Institute of Technology.

CGAL, 2012. Computational Geometry Algorithms Library (release 4.1). http://www.cgal.org.

Desbrun, M., KAnso, E., And Tong, Y. 2007. Discrete differential forms for computational modeling. In Discrete Differential Geometry, A. Bobenko and P. Schröder, Eds. Springer.

Desbrun, M., Donaldson, R., And Owhadi, H. 2013. Modeling across scales: Discrete geometric structures in homogenization and inverse homogenization. In Multiscale analysis and nonlinear dynamics: from genes to the brain, M. Z. Pesenson, Ed., vol. 8 of Reviews of Nonlinear Dynamics and Complexity. Wiley. Extended version of arXiv:0904.2601 [math.AP], 2009.

Fisher, M., Schröder, P., Desbrun, M., And Hoppe, H. 2007. Design of tangent vector fields. In Proceedings of ACM SIGGRAPH.

Fosdick, R., AND SCHULER, K. 2003. Generalized Airy stress functions. Meccanica 38, 5, 571-578. 
Fraternali, F., Angelillo, M., And Fortunato, A. 2002. A lumped stress method for plane elastic problems and the discretecontinuum approximation. International Journal of Solids and Structures 39, 6211-6240.

FRATERnALI, F. 2010. A thrust network approach to the equilibrium problem of unreinforced masonry vaults via polyhedral stress functions. Mechanics Research Communications 37, 2, 198-204.

FRATERnALI, F. 2011. A mixed lumped stress-displacement approach to the elastic problem of masonry walls. Mechanics Research Communications 38, 176-180.

Giaquinta, M., AND GiUsti, E. 1985. Researches on the equilibrium of masonry structures. Archive for Rational Mechanics and Analysis 88, 359-392.

Glickenstein, D., 2005. Geometric triangulations and discrete Laplacians on manifolds. arXiv.org:math/0508188.

Grady, L. J., And Polimeni, J. R. 2010. Discrete Calculus: Applied Analysis on Graphs for Computational Science. Springer.

Green, A., And Zerna, W. 2002. Theoretical Elasticity. Dover.

HEYMAN, J. 1966. The stone skeleton. International Journal of Solids and Structures 2, 2, 249-279.

MERCAT, C. 2001. Discrete Riemann surfaces and the Ising model. Comm. Math. Phys. 218, 177-216.

MÉRIGOT, Q. 2011. A multiscale approach to optimal transport. Computer Graphics Forum 30, 5, 1583-1592.

Mullen, P., Memari, P., De Goes, F., And Desbrun, M. 2011. HOT: Hodge-optimized triangulations. ACM Trans. Graph. 30, 4 (July), 103:1-103:12.

O'DWYER, D. 1999. Funicular analysis of masonry vaults. Computers \& Structures $73,1-5,187-197$.

Preparata, F. P., And Shamos, M. I. 1985. Computational Geometry: An Introduction. Springer-Verlag.

Tong, Y., Alliez, P., Cohen-Steiner, D., And Desbrun, M. 2006. Designing quadrangulations with discrete harmonic forms. In Symposium on Geometry Processing, 201-210.

Vouga, E., Höbinger, M., Wallner, J., and Pottmann, H. 2012. Design of self-supporting surfaces. ACM Trans. Graph. 31 4, 87:1-87:11.

WÄCHTER, A., AND BIEgler, L. T. 2006. On the implementation of an interior-point filter line-search algorithm for large-scale nonlinear programming. Math. Program. 106, 1, 25-57.

WARdeTZKy, M., MATHUR, S., KÄLBERER, F., AND GRINSPUn, E. 2007. Discrete Laplace operators: no free lunch. In Symposium on Geometry Processing, 33-37.

Whiting, E., Ochsendorf, J., And Durand, F. 2009. Procedural modeling of structurally-sound masonry buildings. ACM Transactions on Graphics 28, 5, 112:1-112:10

Whiting, E., Shin, H., Wang, R., Ochsendorf, J., And DURAND, F. 2012. Structural optimization of 3D masonry buildings. ACM Transactions on Graphics 31, 6, 159:1-159:11.

Wong, Y. W., AND PellegRino, S. 2006. Wrinkled membranes Part II: analytical models. Journal of Mechanics of Materials and Structures 1, 25-59.
Zayer, R., Rossl, C., And SeIdel, H.-P. 2005. Discrete tensorial quasi-harmonic maps. In Proceedings of Shape Modeling and Applications, 278-287.

\section{Appendix}

In this appendix, we provide the analytical expressions of several derivatives used in the paper. Deriving these expressions requires basic trigonometric identities, and patience.

\section{Derivatives w.r.t. $w$ :}

$$
\begin{aligned}
& \partial_{w_{i}} d_{i j}=\frac{1}{2|i j|}, \quad \partial_{w_{i}} d_{j i}=-\frac{1}{2|i j|}, \\
& \partial_{w_{i}} h_{j k i}=\frac{\cot \alpha_{i j k}}{2|i j|}, \partial_{w_{i}} h_{i j k}=\frac{\cot \alpha_{j k i}}{2|i k|}, \partial_{w_{i}} h_{k i j}=-\frac{|j k|}{4|i j k|} .
\end{aligned}
$$

Derivatives w.r.t. $c$ :

$$
\partial_{c_{b}} d_{i j}=\frac{\gamma_{j i}^{b}}{2|i j|}, \quad \partial_{c_{b}} h_{j k i}=\frac{\cot \alpha_{i j k}}{2|i j|} \gamma_{k i}^{b}+\frac{\cot \alpha_{k i j}}{2|i j|} \gamma_{k j}^{b} .
$$

Hessian of $\mathcal{E}$ :

$$
\begin{aligned}
\partial_{w_{i}, w_{j}}^{2} \mathcal{E}= & \frac{\cot \alpha_{k i j}}{2|i j|^{2}}\left(z_{k}-z_{j}\right)+\frac{\cot \alpha_{i j k}}{2|i j|^{2}}\left(z_{k}-z_{i}\right) \\
& +\frac{\cot \alpha_{j i l}}{2|i j|^{2}}\left(z_{l}-z_{j}\right)+\frac{\cot \alpha_{l j i}}{2|i j|^{2}}\left(z_{l}-z_{i}\right), \\
\partial_{w_{i}, w_{i}}^{2} \mathcal{E}= & -\sum_{j \in \mathcal{N}(i)} \partial_{w_{i}, w_{j}}^{2} \mathcal{E}, \\
\partial_{w_{i}, c_{b}}^{2} \mathcal{E}= & \frac{1}{2} \sum_{j \in \mathcal{N}(i)}\left(\frac{z_{i}-z_{j}}{|i j|}\right) \partial_{c_{b}} h_{i j} .
\end{aligned}
$$

We also note that the energy $\mathcal{E}$ has an interesting derivative w.r.t. $c$ :

$$
\partial_{c_{b}} \mathcal{E}=\frac{1}{2} \sum_{i j} \star_{i j}^{\sigma}\left(z_{j}-z_{i}\right) \gamma_{i j}^{b}=\frac{1}{2}\left\langle\mathbf{d}_{0} z, \gamma^{b}\right\rangle_{\sigma},
$$

where $\langle\cdot, \cdot\rangle_{\sigma}$ is the inner product induced by $\sigma$.

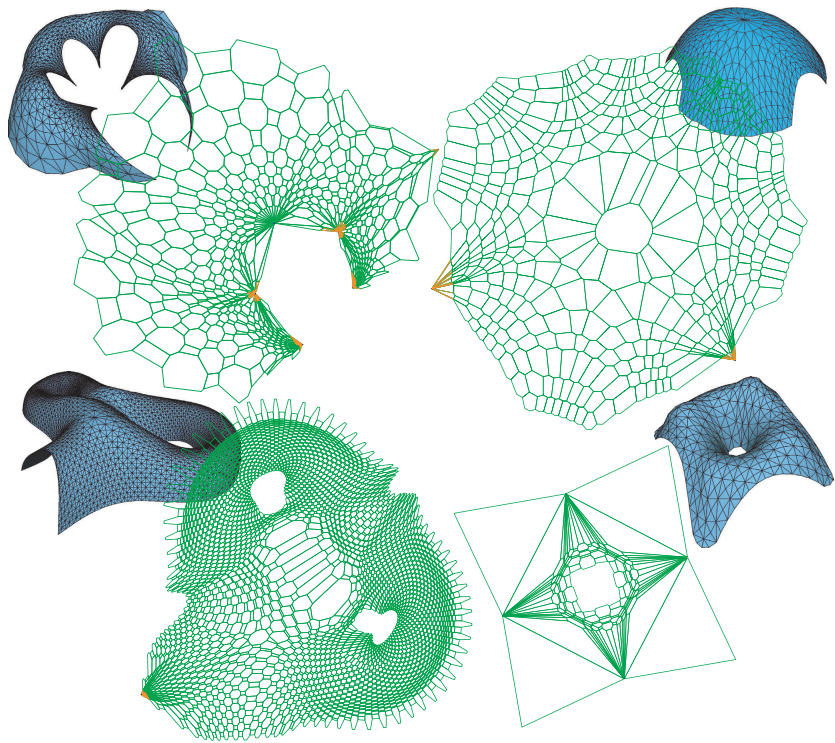

Figure 9: Stress-induced Dual. Self-supporting simplicial surfaces and their stress-induced dual diagrams computed via stress and hole-induced stress optimization (\$4.1 and 4.2) for a series of meshes of arbitrary topology from [Vouga et al. 2012], with boundary normal stresses set to zero $(\tau=0)$. 